\title{
Least order fault and model detection using multi-models
}

\author{
A. Varga
}

\begin{abstract}
Multi-model based fault detection is often a viable alternative to various multi-model based state estimation techniques using banks of Kalman filters. A main advantage of the fault detection techniques based approach is the possibility to use detectors having low order dynamics with disturbance decoupling capabilities. The proposed synthesis algorithm of detectors relies on numerically reliable rational nullspace techniques enhanced with optimal tuning of detection sensitivities. The applicability of the multi-model based approach to solve fault identification problems is illustrated by solving a flight actuator fault detection problem with simultaneous faults.
\end{abstract}

\section{INTRODUCTION}

The multi-model based modelling of physical faults associates to each fault mode of a system a distinct model. Let assume that we have $N>1$ models describing the normal and faulty operation modes of a system, and for $i=1, \ldots, N$, the $i$-th model is specified in the input-output form

$$
\mathbf{y}^{(i)}(\lambda)=G_{u}^{(i)}(\lambda) \mathbf{u}(\lambda)+G_{d}^{(i)}(\lambda) \mathbf{d}(\lambda),
$$

where the $p$-dimensional vector $y^{(i)}(t)$ is the output of the $i$-th system with the $m_{u}$-dimensional control input $u(t)$ and $m_{d}$-dimensional disturbance input $d(t)$, respectively, and $G_{u}^{(i)}(\lambda)$ and $G_{d}^{(i)}(\lambda)$ are the transfer-function matrices (TFMs) from the corresponding plant inputs to outputs. The frequency variable $\lambda$ stays either for the Laplace-transform variable $s$ for a continuous-time system or the Z-transform variable $z$ for a discrete-time system. Bolded notation is used for Laplace- or Z-transformed time vectors.

The state-space realization corresponding to $i$-th model (1) is a generalized (descriptor) state-space model

$$
\begin{aligned}
E^{(i)} \lambda x^{(i)}(t) & =A^{(i)} x^{(i)}(t)+B_{u}^{(i)} u(t)+B_{d}^{(i)} d(t) \\
y^{(i)}(t) & =C^{(i)} x^{(i)}(t)+D_{u}^{(i)} u(t)+D_{d}^{(i)} d(t)
\end{aligned}
$$

where $x^{(i)}(t)$ is the $n_{i}$-dimensional state vector of the $i$ th system and $\lambda$ is the differential or the advance operator, depending on the system type, continuous or discrete, respectively. We will assume that the pencils $A^{(i)}-\lambda E^{(i)}$ are regular. For generality, we will allow for $E^{(i)} \neq I_{n_{i}}$ and even for possibly non-singular $E^{(i)}$, although for most practical applications this generality is not required.

The two model types fulfills different scopes in our presentation. While the input-output description allows a convenient higher level framework to present conceptual approaches in terms of TFMs, the state-space models are exclusively used to develop numerically reliable computational methods.

In many practical applications, the underlying system model is a nonlinear state-space model which depends explicitly on some parameters, say $\theta$. A multi-model with the matrices of components models defined as in (2) can be often interpreted as resulting from repeated trimming and linearization of the underlying nonlinear model for selected values of the parameters $\theta^{(i)}, i=1, \ldots, N$. It follows that the multi-model description is a very convenient way to describe parametric faults (also called multiplicative faults), and, thus practically covers all usual categories of faults.

The multi-model approach to model systems with faults offers a simple framework to solve the fault detection problem (i.e., to detect the presence of a fault) by determining via suitable residual signals which model best fits with the measured outputs and control inputs, and thus identifying the underlying fault. This model detection problem can be solved by adapting the synthesis tools available for standard fault detection problem. An important application of this approach is in solving fault identification problems, i.e., determining the exact nature and size of the underlying fault.

Model detection techniques used for fault identification, are specially well suited when used in conjunction with fault tolerant control schemes [1], where reconfiguration actions take place after detecting a specific faulty situation. Similarly, model detection can become a real alternative to Kalman filter based approaches [2], [3] used for switching or interpolating among different controllers in multi-model adaptive control or in interacting multiple model (IMM) Kalman filters based reconfiguration schemes [4]. The main potential advantages of using model detection filters over various Kalman-filter based techniques are the ability of formers to exactly decouple the influence of non-stochastic disturbances from the residual signals and their significantly lower dynamical orders.

In this paper we propose a general synthesis procedure of least order model detection filters with disturbance decoupling capabilities. The proposed procedure relies on numerically reliable rational nullspace techniques developed for the synthesis of residual generators for fault detection [5], [6]. By exploiting the existing parametric freedom in the synthesis problem, the proposed procedure can be enhanced to provide an optimal tuning of detection sensitivities. The applicability of the multi-model based approach to solve fault identification problems is illustrated by a flight actuator fault detection problems with simultaneous faults. The low orders of the resulting detectors allow to increase the number of employed detectors such that a satisfactory identification of fault sizes is guaranteed. This aspect is of paramount importance for the applicability of multi-model techniques.

\section{Model Detection}

Assume we have $N$ models describing the normal and faulty operations of a system, and for $i=1, \ldots, N$ the $i$-th 
model is specified in the input-output form (1). The model detection problem (MDP) can be formulated as follows: determine $N$ stable and proper residual generator filters

$$
\mathbf{r}^{(i)}(\lambda)=Q^{(i)}(\lambda)\left[\begin{array}{l}
\mathbf{z}(\lambda) \\
\mathbf{u}(\lambda)
\end{array}\right], i=1, \ldots, N
$$

such that for all $u(t)$ and $d(t)$ we have:

(i) $r^{(i)}(t)=0$ when $z(t)=y^{(i)}(t)$;

(ii) $r^{(i)}(t) \neq 0$ when $z(t)=y^{(j)}(t)$ for $i \neq j$;

(iii) $r^{(i)}(t)$ is asymptotically bounded.

This formulation of the MDP allows the detection of the "actual" model by simply looking for the residual signal with the smallest magnitude. If the $N$ faults occur one at a time, this approach ensures the fault detection and isolation by identifying the actual model describing the faulty situation.

The requirements formulated for the MDP can be easily expressed in equivalent algebraic design conditions for each of the $N$ residual generators. To simplify the presentation, we denote

$$
G^{(i)}(\lambda):=\left[\begin{array}{cc}
G_{u}^{(i)}(\lambda) & G_{d}^{(i)}(\lambda) \\
I_{m_{u}} & 0
\end{array}\right]
$$

The decoupling condition $(i)$ is then equivalent to determine each detector $Q^{(i)}(\lambda)$ such that

$$
Q^{(i)}(\lambda) G^{(i)}(\lambda)=0
$$

The detectability condition $(i i)$ enforces the unambiguous detection of the $i$-th model by additionally requiring that for all $j \neq i$

$$
R^{(i, j)}(\lambda):=Q^{(i)}(\lambda) G^{(j)}(\lambda) \neq 0 .
$$

In what follows we call $R^{(i, j)}(\lambda)$ residual models. To fulfill requirement $(i i i)$, each detector $Q^{(i)}(\lambda)$ must additionally ensure that all $R^{(i, j)}(\lambda)$ are stable TFMs. This requirement is automatically fulfilled by any stable and proper detector if each component system (1) is stable.

The number of outputs $q_{i}$ of the filter $Q^{(i)}(\lambda)$ can be chosen arbitrarily between 1 and $p-r_{d}^{(i)}$, where $r_{d}^{(i)}:=$ $\operatorname{rank} G_{d}^{(i)}(\lambda)$. Generally the least order dynamics can be achieved with $q_{i}=1$. However, higher values of $q_{i}$ provide occasionally a better sensitivity condition (see later) for model detection.

The solvability conditions of the MDP are similar to those for the standard fault detection problem (FDP) and result by transcribing appropriately the conditions (5) and (6).

Theorem 1: For the multi-model system defined by the component systems (1), the MDP is solvable if and only if for each $i, j=1, \ldots, N, i \neq j$, we have

$$
\operatorname{rank}\left[G_{d}^{(i)}(\lambda) G_{d}^{(j)}(\lambda) G_{u}^{(i)}(\lambda)-G_{u}^{(j)}(\lambda)\right]>\operatorname{rank} G_{d}^{(i)}(\lambda)
$$

Provided the MDP is solvable, each residual generation filter $Q^{(i)}(\lambda)$ can be determined by stacking up to $q_{\max }$ row vectors representing linear combinations of vectors lying in the left nullspace of $G^{(i)}(\lambda)$, where $q_{\max }$ is an given upper bound on the number of detector outputs. The synthesis procedure of least order detectors is similar to that one of fault detection filters [5]:

\section{Procedure: Synthesis of a Bank of Residual Generators} for Model Detection

Inputs: $q_{\max }, G_{u}^{(j)}(\lambda), G_{d}^{(j)}(\lambda)$, for $j=1, \ldots, N$;

Outputs: $Q^{(i)}(\lambda), q_{i} \leq q_{\max }$, for $i=1, \ldots, N$;

$R^{(i, j)}(\lambda)$ for $i, j=1, \ldots, N$.

For $i=1, \ldots, N$

1) Compute a minimal proper basis $Q^{(i)}(\lambda)$ for the left nullspace of $G^{(i)}(\lambda)$ defined in (4); compute $R^{(i, j)}(\lambda)=Q^{(i)}(\lambda) G^{(j)}(\lambda)$ for $j=1, \ldots, N$.

2) If $R^{(i, j)}(\lambda)=0$ for any $j \neq i$, Exit (no solution exists).

3) Choose a $q_{i} \times(p+m)$ rational matrix $W(\lambda)$ with $q_{i}=\min \left\{q_{\max }, p-r_{d}^{(i)}\right\}$, such that $W(\lambda) Q^{(i)}(\lambda)$ has least McMillan degree and $W(\lambda) R^{(i, j)}(\lambda) \neq$ 0 for $j=1, \ldots, N, j \neq i$; compute $Q^{(i)}(\lambda) \leftarrow$ $W(\lambda) Q^{(i)}(\lambda)$ and $R^{(i, j)}(\lambda) \leftarrow W(\lambda) R^{(i, j)}(\lambda)$ for $j=1, \ldots, N, j \neq i$.

4) Choose a $q_{i} \times q_{i}$ rational matrix $M(\lambda)$ such that $M(\lambda) Q^{(i)}(\lambda)$ has a desired stable dynamics and $M(\lambda) R^{(i, j)}(\lambda)$ for $j=1, \ldots, N, j \neq i$ are stable; compute $Q^{(i)}(\lambda) \leftarrow M(\lambda) Q^{(i)}(\lambda)$ and $R^{(i, j)}(\lambda) \leftarrow$ $M(\lambda) R^{(i, j)}(\lambda)$ for $j=1, \ldots, N, j \neq i$.

The computational algorithms underlying this procedure employ state-space representations of component models as in (2) and are described in Section III. In what follows, we shortly discuss the ideas behind the determination of detectors with least dynamical orders at Step 3) and a possible enhancement of the synthesis procedure by using an optimization-based tuning of existing free parameters in the least order detectors.

To determine detectors with least dynamical orders at Step 3), a systematic search over candidate detectors of increasing orders has to be performed. This search involves two stages, which are repeatedly performed. In the first stage, a suitable candidate detector with least possible order is formed as a set of $q_{i}$ (usually randomly chosen) linear combinations of the left nullspace basis vectors determined at Step 1). This detector has the generic form $W(\lambda) Q^{(i)}(\lambda)$, where each of the $q_{i}$ rows of $W(\lambda)$ can be interpreted as a rational vector whose components are the scalar rational functions used to build the linear combinations of basis vectors. The details of this stage are explained in the next section. Then, in the second stage, the detectability conditions (6) are checked for the candidate detector. If the check fails, both stages are performed again with a new candidate detector of higher order. The iterations are continued until an admissible detector is found. The final orders of individual detectors can occasionally further increase at Step 4) if the cancellation of unstable poles in the component models is necessary, in accordance with requirement $(i i i)$.

After an admissible detector of least order is determined, instead of using randomly chosen linear combinations, it is possible to choose the existing free parameters such that a 
certain sensitivity condition for each residual is minimized. We can define such a sensitivity condition of a residual to individual models by extending the similar concept introduced for fault detection in [7] for the sensitivity condition of a residual to faults. Consider the $i$-th residual generator $Q^{(i)}(\lambda)$ and the corresponding residual models $R^{(i, j)}(\lambda)$ defined in (6). We define the sensitivity condition of $i$-th residual as

$$
\xi_{i}:=\max _{j}\left\|R^{(i, j)}(\lambda)\right\|_{\infty} / \min _{j \neq i}\left\|R^{(i, j)}(\lambda)\right\|_{\infty}
$$

This definition allows to interpret $\xi_{i}$ as a measure of the largest relative distance from model $i$ to the rest of models. To see that, we use (5) to alternatively express $R^{(i, j)}(\lambda)$ as

$R^{(i, j)}(\lambda)=Q^{(i)}(\lambda)\left[\begin{array}{cc}G_{u}^{(j)}(\lambda)-G_{u}^{(i)}(\lambda) & G_{d}^{(j)}(\lambda)-G_{d}^{(i)}(\lambda) \\ 0 & 0\end{array}\right]$

Thus, $\left\|R^{(i, j)}(\lambda)\right\|_{\infty}$ is a measure of the weighted distance (or gap) between the models $i$ and $j$. It follows that $\xi_{i}$ can be interpreted as the largest relative distance (largest gap over the smallest gap) from model $i$ to all other models.

The importance of having smaller sensitivity conditions lies in the fact that a large value of the sensitivity condition indicates potential difficulties in detecting reliably adjacent models to a given set of component models. By selecting the least order detector such that its free parameters are optimally determined to minimize the sensitivity condition of residuals to individual models appears to be very useful to enhance the detection robustness. As it will be apparent in the example presented in Section IV, the proposed approach can tremendously enhance the reliability of the detection of adjacent models. The precise meaning of the free parameters to be optimally tuned at Steps 3) and 4) are described in the next section.

\section{Computational Algorithms}

In this section we discuss shortly the computational algorithms underlying the synthesis procedure presented in the previous section.

\section{A. Computation of minimal proper bases}

For the computation of a minimal proper basis $Q^{(i)}(\lambda)$ for the left nullspace of $G^{(i)}(\lambda)$ defined in (4) we employ the pencil method proposed in [8] based on the state-space realization (2). This method exploits the simple fact that $Q^{(i)}(\lambda)$ is a left nullspace basis of $G^{(i)}(\lambda)$ iff $\left[M^{(i)}(\lambda) \mid Q^{(i)}(\lambda)\right]$ is a left nullspace basis of the correspondingly partitioned system matrix

$$
S^{(i)}(\lambda)=\left[\begin{array}{ccc}
A^{(i)}-\lambda E^{(i)} & B_{u}^{(i)} & B_{d}^{(i)} \\
\hline C^{(i)} & D_{u}^{(i)} & D_{d}^{(i)} \\
0 & I_{m_{u}} & 0
\end{array}\right] .
$$

Thus, to compute $Q^{(i)}(\lambda)$ we can determine equivalently a left nullspace basis $Y^{(i)}(\lambda)$ for $S^{(i)}(\lambda)$ and then $Q^{(i)}(\lambda)$ simply results as

$$
Q^{(i)}(\lambda)=Y^{(i)}(\lambda)\left[\begin{array}{c}
0 \\
I_{p+m_{u}}
\end{array}\right]
$$

$Q^{(i)}(\lambda)$ can be computed by employing linear pencil reduction algorithms based on orthogonal transformations. Let $U^{(i)}$ and $V^{(i)}$ be orthogonal matrices (for instance, determined by using the algorithms of [9], [10]) such that the transformed pencil $\widetilde{S}^{(i)}(\lambda):=U^{(i)} S^{(i)}(\lambda) V^{(i)}$ is in the Kronecker-like staircase form

$$
\widetilde{S}^{(i)}(\lambda)=\left[\begin{array}{cc}
A_{r}^{(i)}-\lambda E_{r}^{(i)} & A_{r, l}^{(i)}-\lambda E_{r, l}^{(i)} \\
\hline 0 & A_{l}^{(i)}-\lambda E_{l}^{(i)} \\
\hline 0 & C_{l}^{(i)}
\end{array}\right],
$$

where the descriptor pair $\left(A_{l}^{(i)}-\lambda E_{l}^{(i)}, C_{l}^{(i)}\right)$ is observable, $E_{l}^{(i)}$ is non-singular, and $A_{r}^{(i)}-\lambda E_{r}^{(i)}$ has full row rank excepting possibly a finite set of values of $\lambda$ (i.e, the invariant zeros of $S^{(i)}(\lambda)$ ). It follows that we can choose the left nullspace $\widetilde{Y}^{(i)}(\lambda)$ of $\widetilde{S}^{(i)}(\lambda)$ in the form

$$
\tilde{Y}^{(i)}(\lambda)=\left[0\left|C_{l}^{(i)}\left(\lambda E_{l}^{(i)}-A_{l}^{(i)}\right)^{-1}\right| I\right] .
$$

Then, a left nullspace of $G^{(i)}(\lambda)$ is

$$
Q^{(i)}(\lambda)=\widetilde{Y}^{(i)}(\lambda) U^{(i)}\left[\begin{array}{c}
0 \\
I_{p+m_{u}}
\end{array}\right]
$$

If we partition

$$
U^{(i)}\left[\begin{array}{c}
0 \\
I_{p+m_{u}}
\end{array}\right]=\left[\begin{array}{c}
B_{r, l}^{(i)} \\
B_{l}^{(i)} \\
D_{l}^{(i)}
\end{array}\right]
$$

in accordance with the column partitioning of $\widetilde{Y}^{(i)}(\lambda)$ in (9), we obtain from (10)

$$
Q^{(i)}(\lambda)=C_{l}^{(i)}\left(\lambda E_{l}^{(i)}-A_{l}^{(i)}\right)^{-1} B_{l}^{(i)}+D_{l}^{(i)}
$$

or equivalently

$$
Q^{(i)}(\lambda)=\left[\begin{array}{c|c}
A_{l}^{(i)}-\lambda E_{l}^{(i)} & B_{l}^{(i)} \\
\hline C_{l}^{(i)} & D_{l}^{(i)}
\end{array}\right]
$$

which is a descriptor system representation of $Q^{(i)}(\lambda)$. Note that, to obtain this nullspace basis, we performed exclusively orthogonal transformations on the system matrices. We can prove that all computed matrices are exact for a slightly perturbed original system. It follows that the algorithm to compute the nullspace basis is numerically backward stable.

The computation of $R^{(i, j)}(\lambda)=Q^{(i)}(\lambda) G^{(j)}(\lambda)$ at Step 1) can not be done in general by means of explicit realizations. To obtain minimal state space realizations of each $R^{(i, j)}(\lambda)$, the orthogonal controllability/observability staircase based methods of [11] can be used.

\section{B. Computation of least order detectors}

Let $Q^{(i)}(\lambda)$ be the $\left(p-r_{d}^{(i)}\right) \times\left(p+m_{u}\right)$ left proper nullspace basis of $G^{(i)}(\lambda)$ constructed in (11), where we recall $r_{d}^{(i)}=\operatorname{rank} G_{d}^{(i)}(\lambda)$. The computation of a least order detector at Step 3) can be done by solving the following computational problem: given $q_{i}$, the number of desired 
detector outputs, and a $q_{i} \times\left(p-r_{d}^{(i)}\right)$ matrix $H^{(i)}$, determine the output injection matrix $K^{(i)}$ such that the TFM

$$
W(\lambda):=\left[\begin{array}{c|c}
A_{l}^{(i)}+K^{(i)} C^{(i)}-\lambda E_{l}^{(i)} & K^{(i)} \\
\hline H^{(i)} C_{l}^{(i)} & H^{(i)}
\end{array}\right]
$$

and thus also

$W(\lambda) Q^{(i)}(\lambda)=\left[\begin{array}{c|c}A_{l}^{(i)}+K^{(i)} C^{(i)}-\lambda E_{l}^{(i)} & \left.B_{l}^{(i)}+K^{(i)} D_{l}^{(i)}\right) \\ \hline H^{(i)} C_{l}^{(i)} & H^{(i)} D_{l}^{(i)}\end{array}\right]$

have the least McMillan degree and $W(\lambda) R^{(i, j)}(\lambda) \neq 0$ for all $j \neq i$. To solve this problem, minimal dynamic cover techniques can be employed. This approach has been proposed in [5] for scalar output detectors (i.e., $q_{i}=1$ ), but can be extended in a straightforward way to detectors with many outputs.

Computational procedures of minimal dynamic covers are presented in [12]. The general idea of the cover algorithms is to perform a preliminary orthogonal similarity transformation on the system matrices in (11) by applying a special version of the controllability staircase form algorithm (see for example [13]) to the descriptor pair

$$
\left(\left(A_{l}^{(i)}-\lambda E_{l}^{(i)}\right)^{T},\left[\left(H^{(i)} C_{l}^{(i)}\right)^{T}\left(C_{l}^{(i)}\right)^{T}\right]\right)
$$

and then with additional block permutations and nonorthogonal block row/column transformations, the transformed system matrices are put in a special form which allows to cancel the maximum number of unobservable poles. For the so-called Type I dynamic covers [14], two nonsingular transformation matrices $\widehat{U}$ and $\widehat{V}$ result such that

$$
\begin{gathered}
\widehat{U}\left(A_{l}^{(i)}-\lambda E_{l}^{(i)}\right) \widehat{V}=\left[\begin{array}{c|c}
\widehat{A}_{11}-\lambda E_{11} & \widehat{A}_{12}-\lambda E_{12} \\
\hline \widehat{A}_{21} & \widehat{A}_{22}-\lambda E_{22}
\end{array}\right], \\
\widehat{U} B_{l}^{(i)}=\left[\frac{\widehat{B}_{1}}{\widehat{B}_{2}}\right], \quad\left[\frac{C_{l}^{(i)}}{\mid H^{(i)} C_{l}^{(i)}}\right] \widehat{V}=\left[\begin{array}{c|c}
\widehat{C}_{11} & \widehat{C}_{12} \\
\hline 0 & \widehat{C}_{22}
\end{array}\right],
\end{gathered}
$$

where the pairs $\left(\widehat{A}_{11}-\lambda E_{11}, \widehat{C}_{11}\right)$ and $\left(\widehat{A}_{22}-\lambda E_{22}, \widehat{C}_{22}\right)$ are observable, and the submatrices $\widehat{C}_{11}$ and $\widehat{A}_{21}$ have the particular structure

$$
\left[\begin{array}{l}
\widehat{A}_{21} \\
\widehat{C}_{11}
\end{array}\right]=\left[\begin{array}{ll}
0 & A_{21} \\
0 & C_{11}
\end{array}\right]
$$

with $C_{11}$ having full column rank. By taking

$$
K^{(i)}=\widehat{V}\left[\begin{array}{c}
0 \\
K_{2}
\end{array}\right]
$$

with $K_{2}$ satisfying $K_{2} C_{11}+A_{21}=0$, we annihilate $\widehat{A}_{21}$, and thus make all eigenvalues of $\widehat{A}_{11}-\lambda E_{11}$ unobservable. The resulting $W(\lambda) Q^{(i)}(\lambda)$ of least McMillan degree, obtained by deleting the unobservable part, has the minimal state space realization

$$
W(\lambda) Q^{(i)}(\lambda)=\left[\begin{array}{c|c}
\widehat{A}_{22}+K_{2} \widehat{C}_{12}-\lambda E_{22} & \widehat{B}_{2}+K_{2} D_{l} \\
\hline \widehat{C}_{22} & H^{(i)} D_{l}
\end{array}\right]
$$

The resulting least order depends of the choice of $H^{(i)}$. An admissible choice is one which guarantees that $W(\lambda) R^{(i, j)}(\lambda) \neq 0$ for all $j \neq i$. A systematic search for an admissible $H^{(i)}$ must take into account the known achievable dimensions. These are among the possible dimensions of the controllability subspaces of the dual pair $\left(\left(A_{l}^{(i)}-\lambda E_{l}^{(i)}\right)^{T},\left(C_{l}^{(i)}\right)^{T}\right)$, and can be achieved by appropriate choices of $H^{(i)}$. The choice of $H^{(i)}$ for scalar output detectors has been described in [5] and exploits the underlying structure of matrices resulting in the Kroneckerlike form (8). $H^{(i)}$ is chosen with a suitable structure which leads to a certain achievable order (i.e., with a structurally fixed pattern of zeros and nonzero elements) and then the non-zero elements are filled with randomly generated values. A similar strategy can be employed also for $q_{i}>1$, but this aspect will not be pursued here because of lack of space. Instead of using random values, one possibility to enhance the choice of $H^{(i)}$ is by using an optimal choice which minimizes the sensitivity conditions defined in (7).

\section{Assigning a desired stable dynamics}

The computation at Step 4) can be performed using standard left coprime factorization techniques [15]. The basic operation is to determine an appropriate output injection $L^{(i)}$ such that the pair $\left(\widehat{A}_{22}+K_{2} \widehat{C}_{12}+L^{(i)} \widehat{C}_{22}, E_{22}\right)$ has desired generalized eigenvalues. Then, using the realization (12) for the updated $Q^{(i)}(\lambda)$ at Step 3), the factor $M(\lambda)$ results as

$$
M(\lambda)=\left[\begin{array}{c|c}
\widehat{A}_{22}+K_{2} \widehat{C}_{12}+L^{(i)} \widehat{C}_{22}-\lambda E_{22} & L^{(i)} \\
\hline \widehat{C}_{22} & I_{q_{i}}
\end{array}\right]
$$

and $M(\lambda) Q^{(i)}(\lambda)$ is obtained using standard left coprime factorization formulas [15]. A more sophisticated numerically reliable procedure to determine simultaneously $M(\lambda)$ and $M(\lambda) Q^{(i)}(\lambda)$ is based on a sequential pole assignment based approach [16].

\section{EXAMPLE}

We consider the multi-model based solution of a challenging fault identification problem to illustrate the effectiveness of the optimal tuning of free parameters of model detectors. The underlying multi-model has been used in [4] to address a fault tolerant control problem via an IMM-filters based approach. In our study, we focus only on the model detection aspects and show that our approach is a viable alternative both in terms of real-time computational costs as well as the ability to reliably detect adjacent models.

The fault-free state space model describes the lateral dynamics of a F-16 aircraft with the matrices

$$
\begin{aligned}
A^{(1)} & =\left[\begin{array}{cccc}
-0.4492 & 0.0460 & 0.0053 & -0.9926 \\
0 & 0 & 1.0000 & 0.0067 \\
-50.8436 & 0 & -5.2184 & 0.7220 \\
16.4148 & 0 & 0.0026 & -0.6627
\end{array}\right] \\
B_{u}^{(1)} & =\left[\begin{array}{cc}
0.0004 & 0.0011 \\
0 & 0 \\
-1.4161 & 0.2621 \\
-0.0633 & -0.1205
\end{array}\right], C^{(1)}=I_{4}, D_{u}^{(1)}=0
\end{aligned}
$$


The four state variables are the sideslip angle, roll angle, roll rate and yaw rate, and the two input variables are the aileron deflection and rudder deflection. The individual failure models correspond to different levels of surface efficiency degradation. We consider altogether $N=25$ models on a two-dimensional parameter grid of $(\alpha, \beta)$ values defined by the discrete set of points $\{0,0.25,0.5,0.75,1\}$. The component system matrices in (2) are defined for $i=$ $1,2, \ldots, 25$ as: $E^{(i)}=I_{4}, A^{(i)}=A^{(1)}, C^{(i)}=C^{(1)}$, and

$$
B_{u}^{(i)}=B_{u}^{(1)}\left[\begin{array}{cc}
1-\alpha_{i} & 0 \\
0 & 1-\beta_{i}
\end{array}\right],
$$

where $\left(\alpha_{i}, \beta_{i}\right)$ are the values of parameters $(\alpha, \beta)$ on the chosen grid. For example, $\left(\alpha_{1}, \beta_{1}\right)=(0,0)$ corresponds to the fault free situation, while $\left(\alpha_{25}, \beta_{25}\right)=(1,1)$ corresponds to complete failure of the control surfaces.

As basis for comparisons, we designed a first set of 25 scalar output detectors, with least dynamical orders (24 detectors of order 1, 1 detector of order 0 ), with randomly chosen free parameters in $H^{(i)}$ and with all poles assigned to -1 . The resulting norms of residual models for this initial design are shown Fig. 1 and indicate large sensitivity conditions: 190 for $r^{(1)}, 123$ for $r^{(11)}$, and 400 for $r^{(21)}$.

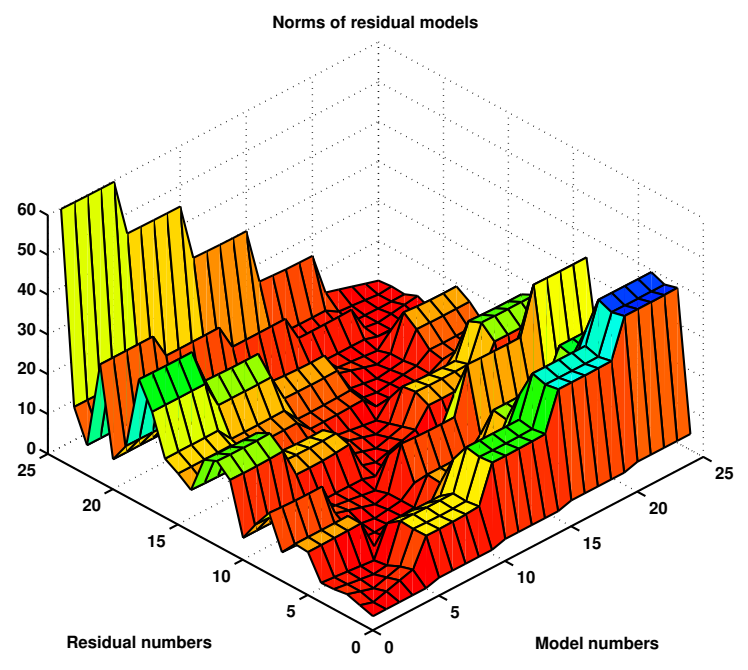

Fig. 1. Norms of residual models - initial design

On a finer grid of the damage parameters of only 0.05 , we determined an estimation of the false detection rate. For each of the 441 parameter combinations we determined the nearest parameter on the coarse grid which thus defines the expected model to be detected. The detected actual model corresponds to the nearest norm in Fig. 1 (which presumably leads to the smallest residual norm). In Fig. 2 we compare the number of matches between the expected and actual models. As it can be observed, there are large deviations between the "expected" models and the detected "actual" models. The number of false decisions divided by the total number of samples can be seen as an empirical measure of the false detection rate. For the initial design this rate was 0.74 , which is unacceptably high. The models with indices 1 (no faults),
11 and 21 were strongly favored by the norm based decision process. Interestingly, these are also the indices of residuals having the largest sensitivity conditions.

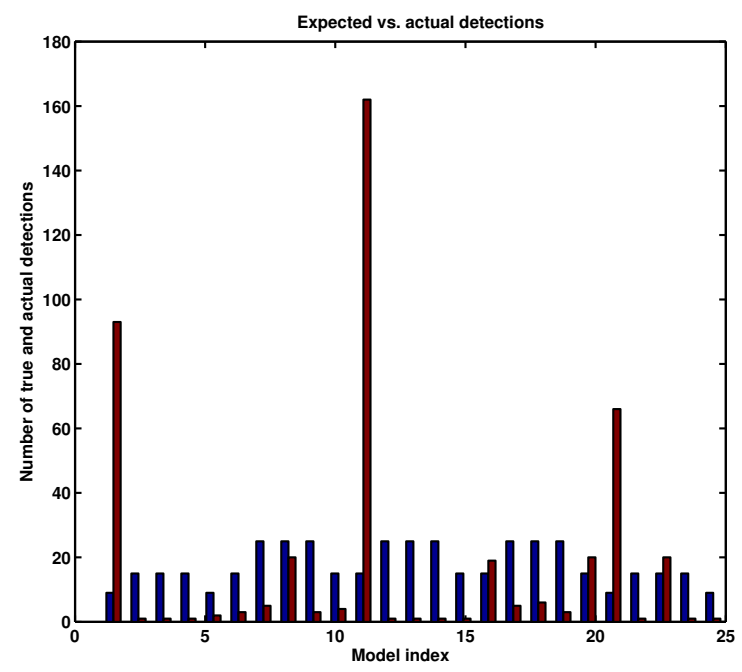

Fig. 2. Expected versus actually detected models - initial design

In a second design, we determined detectors with optimal values of $H^{(i)}$ which minimize the sensitivity conditions of each residual. For convenience, all residuals have been scaled such that the corresponding norms of residual models in Fig. 3 have least nonzero values of unity. The achieved maximum sensitivity condition has been reduced with more than 60 times (from about 400 to about 6). There is a sharp-cut valley along the zero values which are bordered by unity values and this can be also seen in Fig. 4, where the level curves of the norms are plotted. This characteristic shape is an indication of potentially improved detection capabilities of the new set of detectors, because only the values bellow this unity border are instrumental for model detection.

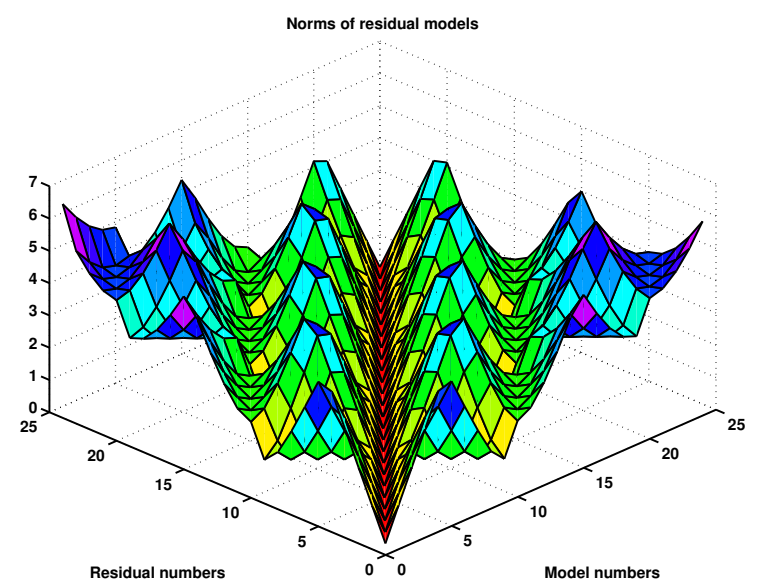

Fig. 3. Norms of residual models - optimal design

In Fig. 5 we compare the number of matches for each model. As it can be observed, there is a perfect match between the "expected" models and the "actual" models, and 


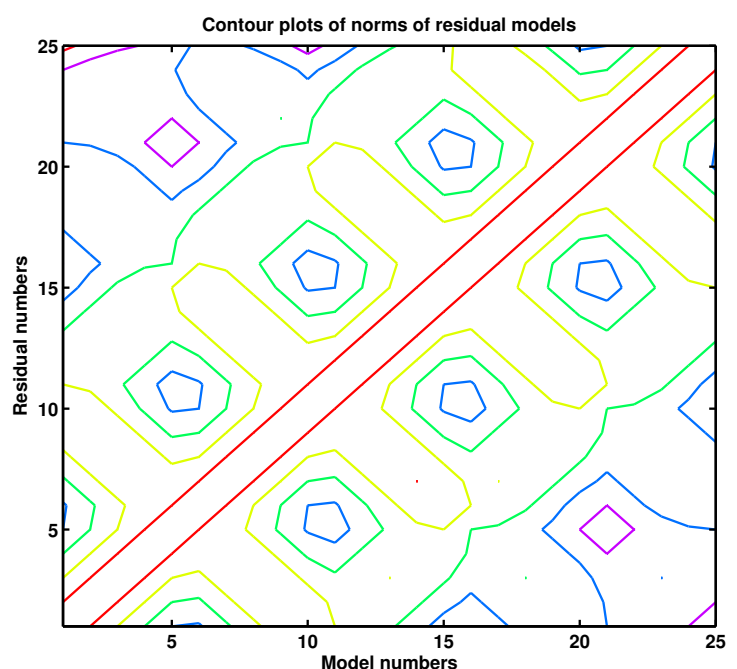

Fig. 4. Contour plots of norms - optimal design

therefore for the optimal design the false decision rate was zero. However, it can be expected that on a finer grid, a number of "false" decisions may result from values lying at the separation boundary between two nearby models. Therefore, these are in fact no false decisions at all.

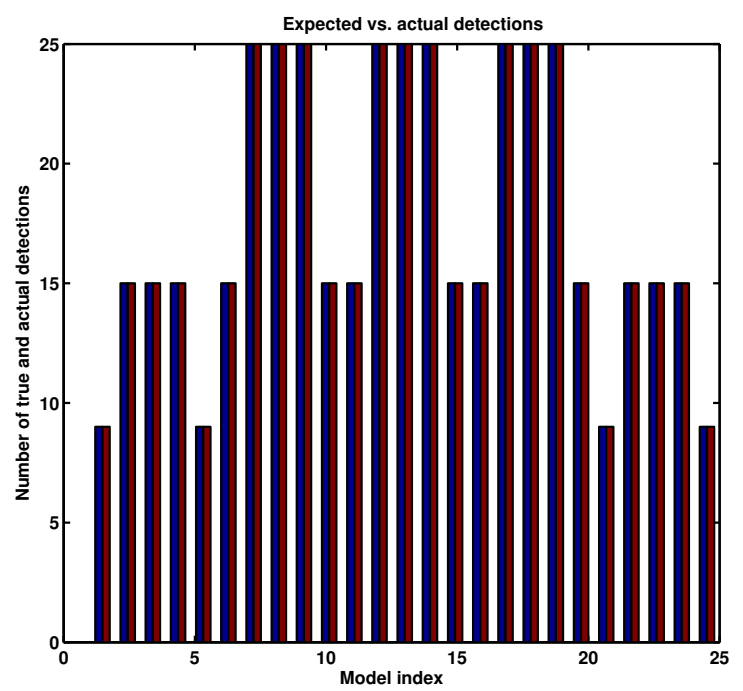

Fig. 5. Expected versus actually detected models - optimal design

\section{CONCLUDING REMARKS}

The two main advantages of using model detection filters are their ability to decouple the influence of non-stochastic disturbances from the residuals and their lower dynamical orders. The latter advantage is illustrated in the employed example: the possibility of using very low order detectors instead of full order Kalman filters. In direct comparison with a Kalman-filter based approach, the total order of the employed detectors is 24, while the Kalman filter based approach would require a total order of $25 \times 4=100$. A third advantage follows from the possibility to assimilate parametric variations with parametric faults. In this way, model detection techniques can easily ensure a certain robustness of model detection schemes. In light of above, model detection could become a real alternative to using banks of Kalman filters [2], [3] used for switching or interpolating among different controllers in multi-model adaptive control schemes.

There are several aspects not discussed in this paper, which could be important in practical applications. One aspect is to exploit intrinsic problem features, as for example, components models which share the same output, state and descriptor matrices, and use explicit realizations of the residual models $R^{(i, j)}(\lambda)$ as derived in [6]. Another aspect is to extend the concept of strong detectability of classes of persistent faults [17] to the model detection problem formulated in this paper. Also model detection in the presence of input and measurement noise can be addressed along the techniques proposed in [18]. And, finally, even the approximate model detection can be an issue in the case when no exact decoupling of disturbances is possible.

\section{REFERENCES}

[1] J. Boskovic and R. Mehra, "A multiple model-based reconfigurable flight control system design," Proc. IEEE CDC, Tampa, Florida. IEEE, December 1998.

[2] K.S.Narendra and J.Balakrishnan, "Adaptive control using multiple models," IEEE Trans. Automat. Control, vol. 42, no. 2, pp. 171-187, 1997.

[3] P. S. Maybeck, "Multiple model adaptive algorithms for detecting and compensating sensor and actuator/surface failures in aircraft flight control systems," Int. J. of Robust and Nonlinear Control, vol. 9, no. 14, pp. 1051-1070, 1999.

[4] S. Kim, J. Choi, and Y. Kim, "Fault detection and diagnosis of aircraft actuators using fuzzy-tuning IMM filter," IEEE Transactions on Aerospace and Electronic Systems, vol. 44, pp. 940-952, 2008.

[5] A. Varga, "On designing least order residual generators for fault detection and isolation," Proc. 16th Internat. Conf. on Control Systems and Computer Science, Bucharest, Romania, 2007, pp. 323-330.

[6] —_ "On computing nullspace bases - a fault detection perspective," Proc. IFAC 2008 World Congress, Seoul, Korea., 2008, pp. 6295-6300.

[7] J. Gertler, Fault Detection and Diagnosis in Engineering Systems. New York: Marcel Dekker, 1998.

[8] A. Varga, "On computing least order fault detectors using rational nullspace bases," Proc. of IFAC Symp. SAFEPROCESS'2003, Washington D.C., 2003.

[9] T. Beelen, "New algorithms for computing the kronecker structure of a pencil with applications to systems and control theory," Ph. D. Thesis, Eindhoven University of Technology, 1987.

[10] A. Varga, "Computation of Kronecker-like forms of a system pencil: Applications, algorithms and software," Proc. CACSD'96 Symposium, Dearborn, MI, 1996, pp. 77-82.

[11] — , "Computation of irreducible generalized state-space realizations," Kybernetika, vol. 26, pp. 89-106, 1990.

[12] _ - "Reliable algorithms for computing minimal dynamic covers for descriptor systems," Proc. of MTNS'04, Leuven, Belgium, 2004.

[13] — - "Computation of irreducible generalized state-space realizations," Kybernetika, vol. 26, pp. 89-106, 1990.

[14] G. Kimura, "Geometric structure of observers for linear feedback control laws," IEEE Trans. Automat. Control, vol. 22, pp. 846-855, 1977.

[15] K. Zhou, J. C. Doyle, and K. Glover, Robust and Optimal Control. Prentice Hall, 1996.

[16] A. Varga, "Computation of coprime factorizations of rational matrices," Lin. Alg. \& Appl., vol. 271, pp. 83-115, 1998.

[17] J. Chen and R. J. Patton, Robust Model-Based Fault Diagnosis for Dynamic Systems. Kluwer Academic Publishers, London, 1999.

[18] R. Nikoukhah, "Innovations generation in the presence of unknown inputs: application to robust failure detection," Automatica, vol. 30, pp. 1851-1867, 1994. 